\title{
Análisis del impacto de un programa deportivo en niños con Trastorno del Espectro del Autismo Analysis of the impact of a sport program on children with Autism Spectrum Disorder *José María López-Díaz, **Ricardo Moreno-Rodríguez, **José Luis López-Bastías \\ *Escuela Internacional de Doctorado de la Universidad Rey Juan Carlos (España), **Universidad Rey Juan Carlos (España)
}

\begin{abstract}
Resumen. A través de la presente investigación se plantea el objetivo de desarrollar un programa socio-deportivo para mejorar las habilidades motrices y las habilidades sociales de niños con trastorno del espectro del autismo, además de estudiar las repercusiones que tiene la práctica deportiva en niños con trastorno del espectro del autismo La actividad física y deportiva puede ser un pilar fundamental para lograr alcanzar un pleno desarrollo en los ámbitos personales y sociales. Para ello, se articula un programa socio-deportivo con una duración de ocho meses, en el que participan 15 niños con trastorno del espectro del autismo, divididos en tres grupos homogéneos y seleccionados a través de entrevistas semiestructuradas realizadas a su núcleo familiar, tomando como criterio de inclusión que tuvieran edades comprendidas entre los seis y los 12 años, además de tener un grado de apoyos necesarios uno o dos (según el DSM-5). El impacto del programa se evalúa a través de un sistema de rúbrica cumplimentado por dos observadores a lo largo de todas las sesiones. Los resultados obtenidos se obtienen a través de la comparación de las puntuaciones medias entre ambos observadores, estableciendo las puntuaciones medias de forma mensual y realizando la prueba de significación de Wilcoxon para muestras relacionadas. Los resultados muestran diferencias significativas entre dos momentos de medición. El primero, al inicio del programa, con la finalidad de establecer una línea base sobre la que comparar el segundo momento, al finalizar el programa. También se puede observar una tendencia de mejora a través del tiempo, con un leve estancamiento entre los meses de enero y febrero, y una leve reducción del rendimiento al finalizar el programa.
\end{abstract}

Palabras clave. Trastorno del Espectro Autista (TEA), deporte, fútbol, desarrollo motor, habilidades sociales.

\begin{abstract}
The objective of this research is to develop a social-sport program to improve motor skills and social skills of children with autism spectrum disorder, in addition to studying the repercussions that sports practice has on children with autism disorder. Physical and sports activity can be a fundamental pillar for achieving full development in personal and social fields. To this end, a social-sport program with a duration of eight months is developed, involving 15 children with autism spectrum disorder divided into three homogeneous groups and selected through semi-structured interviews conducted with their family nucleus. Inclusion criteria were being between the ages of six and 12 years old; having degree one or two of necessary support (according to the DSM-5). The impact of the program is evaluated through a rubric system completed by two observers throughout all the sessions. The results are obtained by means of comparison of mean scores between both observers, establishing the mean scores on a monthly basis and performing the Wilcoxon significance test for related samples. The results show significant differences between the two measurement times (the first, at the beginning of the program, in order to establish a baseline against which to compare the second time, at the end of the program). A tendency of improvement can also be observed over time, with a slight stagnation between the months of January and February, and a slight reduction in performance at the end of the program.
\end{abstract}

Key words. Autism spectrum disorder (ASD), sport, football, motor skills, social skills.

\section{Introducción}

El Trastorno del Espectro del Autismo (TEA) se clasifica como categoría diagnóstica dentro de los trastornos del neurodesarrollo (Smith et al., 2007). Esta alteración del neurodesarrollo es diagnosticada en la primera infancia y se mantiene durante el proceso de desarrollo de la persona. En los casos en los que la afectación no involucra a las funciones mentales superiores, puede ser diagnosticado cuando las demandas sociales del entorno superan las capacidades de la persona.

En líneas generales, las manifestaciones del TEA se circunscriben a alteraciones a nivel cognitivo y conductual, produciendo en la mayoría de los casos limitaciones en la autonomía personal, además de un cierto grado de discapacidad (Díez-Cuervo et al., 2005). De manera específica, el TEA se caracteriza por patrones de comportamiento, intereses y actividades restrictivos y repetitivos, así como por limitaciones en la comunicación y en la socialización. Además, se ha observado en diversos estudios que los ni-

Fecha recepción: 23-10-19. Fecha de aceptación: 21-05-20 José María López Díaz jm.lopezd19@gmail.com ños con TEA también presentan déficits en las habilidades motrices básicas. También presentan limitaciones a la hora de participar en el juego simbólico o imaginativo. En el caso de niños normotípicos, generalmente la mayor parte de la actividad física es realizada durante el tiempo de juego; sin embargo, esto no sucede en los niños con TEA. A pesar de que los déficits en las habilidades motrices no conforman uno de los criterios diagnósticos del TEA en el Manual Diagnóstico y Estadístico de los Trastornos Mentales en su quinta edición -en inglés, Diagnostic and Statistical Manual of Mental Disorders (DSM-5)-, suelen prevalecer en los niños con este trastorno (Ohara, Kanejima, Kitamura \& Izawa, 2020). Ya en las primeras descripciones de Leo Kanner sobre el autismo se hacía alusión a estas limitaciones. En diversos estudios se aboga por la presencia de déficits motores, alteraciones en la coordinación, dificultades en el mantenimiento de la postura o en la motricidad fina y gruesa, en comparación con niños normotípicos de la misma edad cronológica (Harris, 2017; Kruger, Silveira, \& Marques, 2019). Además, estudios como los de Kruger et al. (2019) manifiestan en sus investigaciones una correlación positiva entre el nivel de afectación del TEA y las limitaciones en las habilidades motrices, es decir, estas son mayores cuando el nivel de afectación del trastorno es mayor. 
La condición de este trastorno suele influir en la participación de actividades de índole social y física, disminuyendo las oportunidades para iniciar una interacción social y aumentando la predisposición a la inactividad (Ruiz Vicente, et al., 2015). Dicho de otro modo, el deterioro de las habilidades motrices dificulta la participación en actividades físicas grupales, y como consecuencia se pierden oportunidades de socialización en el contexto de la actividad física (Kruger, et al., 2019). En los últimos años se ha incidido en la investigación sobre la relación entre las habilidades motrices y las habilidades de comunicación social en niños con TEA, donde se ha observado que las limitaciones en las habilidades motrices se relacionan con las alteraciones en la comunicación social. Además, se ha comprobado que intervenciones que trabajan el abordaje de habilidades motrices a través de actividad física producen una mejora en la competencia social así como en las habilidades motrices (Healy, Nacario, Braithwaite \& Hopper, 2018; Kruger, et al., 2019; Ohara, et al., 2020). Atendiendo a estos resultados, se hace necesario diseñar e implementar programas basados en la actividad física programada que permitan crear un entorno de juego estructurado, y que sean favorecedores del aumento de posibilidades para participar en actividades físicas así como en interacciones sociales (Wong et al., 2015). Los resultados en otras investigaciones son prometedoras. Por ejemplo, Healy et al. (2018) en su estudio sobre los efectos en las intervenciones grupales centradas en actividad física, concretamente en juegos colectivos, obtuvo una mejora significativa en habilidades relacionadas con la manipulación, habilidades locomotrizs, habilidades relacionadas con la aptitud física (fuerza muscular y resistencia), así como en la competencia social que incluía la comunicación social, el funcionamiento adaptativo y el comportamiento adaptado durante el juego.

\section{Contexto}

El Manual Diagnóstico y Estadístico de los Trastornos Mentales (DSM-5), en su quinta edición, define el TEA como un espectro de trastornos que comparten un conjunto de síntomas basados en, por un lado, déficits persistentes en la comunicación social e interacción social a lo largo de múltiples contextos, y por otro, en patrones repetitivos y restringidos de conductas, actividades e intereses. Además, el DSM5 clasifica el TEA en tres niveles de severidad en función del nivel de apoyo requerido por la persona, siendo el Grado 1, en el que menor nivel de necesidad de apoyo requiere y el grado 3, el que mayor necesidad de apoyo presenta (American Psychiatric Association, 2013).

Actualmente, existen diversas estrategias de trabajo así como tratamientos, como por ejemplo, intervención sensoriomotriz, tratamientos psicoeducativos, intervención psicológica o el tratamiento biomédico, entre otros, que sirven de aplicación como programa base para estudiar y analizar los resultados de mejora en el funcionamiento general de las personas con TEA (Fuentes et al., 2006). No obstante, en los últimos años se ha apostado por otras alternativas de intervención, basadas en la terapia con animales o la intervención basada en la actividad física (Wong, et al., 2015). Asimismo, en este último caso, existen varias estrategias que aseguran la eficacia de la intervención basada en la acti- vidad física dirigida a niños con TEA (Arroyo, 2019). Estas estrategias se concretan en el establecimiento de una estructura predecible para la mejora del comportamiento, la participación entre iguales para conseguir efectos positivos en la comunicación social, la guía y el reforzamiento positivo de comportamientos adecuados para favorecer la repetición de esos comportamientos, la enseñanza para regular y dirigir las emociones y el apoyo en información visual y el uso de tecnología, como contenido audiovisual a través de una tablet, para modelar los comportamientos deseados (Arroyo, 2019).

La actividad física en grupo es considerada una estrategia que guía el diseño de la intervención en personas con TEA. Este enfoque de trabajo se ve motivado por los beneficios que aporta al desarrollo del niño; además, constituye una herramienta a través de la cual se puede abordar de forma transversal dos de las áreas donde existen más dificultades, el desarrollo motor y la competencia social. Por ello, existe cada vez más un amplio interés en emplear el deporte como medida de atención dirigido a personas con discapacidad, con el fin de trabajar no solo el aspecto físico, sino también el ámbito social (Ibáñez \& Mudarra, 2004; Wong et al., 2015; Healy et al., 2018; Ohara et al., 2020).

Atendiendo a Reinders, Branco, Wright, Fletcher y Bryden (2019), la participación y el acceso a programas basados en la actividad física puede verse afectada por la falta de aceptación de las normas sociales establecidas, lo que da lugar a una situación de desigualdad de oportunidades en la participación en el programa basado en la actividad física, en comparación con el grupo neurotípico de referencia. Asimismo, en otro estudio se concluye que los padres informan de la existencia de barreras para participar en actividades deportivas convencionales (May et al., 2018). Conocer las barreras y los facilitadores del TEA es clave para, por un lado, diseñar programas deportivos adaptados a las necesidades de los niños con TEA y, por otro, para asegurar la participación en ellos (Mat et al., 2018). En consecuencia, adquiere mayor importancia la superación de dificultades asociadas a la comunicación social, así como la reducción de los comportamientos estereotipados propios del trastorno que la correcta realización de las actividades físicas. Por este motivo, es importante prestar atención a este ámbito y promover el acceso a actividades físicas teniendo en cuenta las características de este colectivo (Gutiérrez, 2004). Como resultado, la actividad física debe enfocarse, principalmente, desde una perspectiva lúdica. De este modo, es posible asegurar la adhesión a la práctica deportiva grupal (May et al., 2018).

Por su parte, la práctica la actividad física en niños con TEA es menor en comparación con el grupo de referencia neurotípico. Este hecho se explica por escaso número de oportunidades para participar en actividades grupales así como por el mal ajuste de las adaptaciones implementadas para garantizar la participación satisfactoria en la actividad (Holland, Holland, Haegele \& Alber-Morgan, 2019). Uno de los estudios que llevo a cabo Chien-Yu Pan (2008) concluyó que el porcentaje de actividad física realizada era menor en los niños TEA durante el recreo. Esto se debía, principalmente, a la falta de apoyos y adaptaciones que debía tener el menor con TEA para participar en el juego (Pan, 2008). Por su parte, Obrusnikova y Cavalier (2010) evaluaron las barreras 
de la práctica de actividad física en niños TEA después del colegio. Además de no alcanzar el tiempo recomendado en la práctica física, expusieron la dificultad que supone practicar actividad física que implique un alto nivel de coordinación y de motricidad fina o gruesa (Obrusnikova \& Cavalier, 2010).

Howells et al. (2019) destaca la escasez de estudios que indagan sobre el impacto de programas deportivos en equipo. Sin embargo, estos programas favorecen comportamientos prosociales así como mayores oportunidades de interacción social (Cei et al., 2017; May et al., 2018). El fútbol o balompié es uno de los deportes en equipo más populares en España, tanto a nivel mediático como en su práctica lúdica en edad escolar. Surge así una línea de trabajo en la que profundizar en investigación, no solo por los escasos costes económicos o la accesibilidad física al entorno que implica jugar al fútbol, sino porque supone una oportunidad que implica un mayor número de posibilidades de interacción social, aspecto muy relevante en el colectivo de niños con TEA. En un estudio sobre el impacto de un programa de fútbol dirigido a niños con TEA en el funcionamiento social, comportamiento adaptativo y comunicación se obtuvieron resultados muy favorables (Howells et al., 2020) en las variables mencionadas. Asimismo, se observó una disminución de conductas problemáticas así como de sintomatología relacionada con la ansiedad.

Atendiendo a las anteriores aportaciones, este programa dirigido a niños con TEA se basa en actividades físicas basadas en el deporte del fútbol. Otro de los motivos que ha determinado la selección del fútbol reside en su componente de transmisión de valores educativos, que permiten garantizar un entorno que permita al niño enfrentarse a diferentes situaciones superándolas, apoya el desarrollo de su autoestima así como el conocimiento sobre sí mismo (Merino, Jarie \& Usán, 2019). En consecuencia, se debe trabajar de forma intencionada la adquisición de valores positivos (Monjas, Ponce \& Gea, 2015) y esos deben actuar como un recurso que permita la autorregulación, la comprensión del entorno social, la comunicación, con el fin último de abordar las dificultades asociadas al TEA: habilidades sociales, comunicación social y autorregulación emocional. Por su parte, la práctica de actividades físicas basadas en el fútbol requiere de una serie de conceptos y habilidades que se adquieren a través del entrenamiento. A nivel conceptual, el fútbol se compone de una parte teórica y una práctica, además de conocer y seguir una serie de normas que servirán para poner en práctica el deporte y mejorar posteriormente las habilidades motrices trabajadas comúnmente en el fútbol. Este hecho es imprescindible tenerlo muy presente puesto que es clave para el éxito del programa deportivo dirigido a niños con TEA. Poniendo el foco atencional en la vertiente psicológica y social de la práctica deportiva en equipo, a través del fútbol se transmiten una serie de valores que se van adquiriendo durante el transcurso de la propia práctica deportiva. Algunos de estos valores son la tolerancia, la solidaridad, el respeto, el compañerismo, la igualdad, el trabajo en equipo, la comunicación, la empatía y la generosidad, entre otras (Núñez, 2005).

\section{Objetivos del programa}

Los objetivos del programa de actividades socio-depor- tivas se concretan a continuación:

El eje principal sobre el que se sustenta la investigación es desarrollar un programa deportivo para mejorar las habilidades motrices y las habilidades sociales de niños con trastorno del espectro del autismo.

Por su parte, de manera específica se persigue estudiar las repercusiones que tiene la práctica deportiva en niños con trastorno del espectro del autismo.

\section{Planteamiento}

El proyecto deportivo fue diseñado por el grupo de investigación Diversia y financiado por la Fundación Real Madrid. El perfil al que fue dirigido el programa debía cumplir los siguientes criterios de inclusión:

- Edad comprendida entre seis y doce años.

- Diagnóstico de TEA con nivel uno y nivel dos de necesidades de apoyo según el DSM-5 (2013).

Los jugadores fueron seleccionados de manera aleatoria a partir de las respuestas recibidas ante el anuncio de la inminente realización del programa deportivo. Se realizaron entrevistas semiestructuradas de una hora de duración a un total de 21 familias para conocer las necesidades de apoyo de los niños y niñas que se presentasen. De entre todas las entrevistas que se realizaron con las familias, y que cumpliesen el perfil de acceso, finalmente fueron 15 los niños que participaron en el programa deportivo de fútbol repartidos en tres grupos. Cabe destacar que únicamente se presentó una familia con una niña, que no pudo ser seleccionada debido a que no cumplía el perfil de acceso al tener un Grado 3 de apoyos necesarios (DSM-5). Además, las edades de los niños seleccionados estaban entre los seis y los 12 años, siendo la franja de edad de los siete a los ocho años y los 12 , donde se aglutinaban más participantes, cinco y cuatro, respectivamente. Cada grupo se configuró en función de las posibilidades que tenían los padres de los participantes para poder acudir a las sesiones, a la par que se intentó mantener unas condiciones de necesidades de apoyo grupales similares. Dentro de las posibilidades que se dieron, se consiguió mantener a los participantes con mayores necesidades de apoyo en grupos separados, de modo que resultasen tres grupos lo más homogéneos entre sí posible, tal y como se describe en la Tabla 1.

\begin{tabular}{lccc}
$\begin{array}{l}\text { Tabla 1 } \\
\text { Distribución de grupos en función del grado de } \\
\text { Grado de necesidad } \\
\text { de apoyo }\end{array}$ & $\begin{array}{c}\text { Número de } \\
\text { participantes }\end{array}$ & Total \\
\hline \multirow{2}{*}{ Grupo 1 } & Grado 1 & 3 & \\
& Grado 2 & 1 & 5 \\
Grupo 2 & Grado 3 & 1 & \\
& Grado 1 & 3 & 5 \\
Grado 2 & 2 & \\
Grupo 3 & Grado 3 & 0 & 5 \\
Total & Grado 1 & 3 & 5 \\
\hline
\end{tabular}

Para el desarrollo del proyecto, se llevó a cabo un programa de formación y entrenamiento en fútbol con objetivos estandarizados. Dentro de los entrenamientos deportivos se trabajaron conceptos técnicos, tácticos y físicos. Se organizaron sesiones de entrenamiento adaptados a un nivel básico para poder poner en práctica las acciones técnicas de tiro, pase, control y conducción. A nivel táctico se trabajaron acciones vinculadas a la manera y forma de juego. A nivel físico se potenciaron las cualidades físicas básicas y las ha- 
bilidades motrices de salto, giro, desplazamiento, manipulaciones, lanzamientos y recepciones (Cos, Cos, Buenaventura, Pruna \& Ekstrand, 2010).

El programa tuvo una duración de ocho meses comenzando el programa en el mes de noviembre y finalizando en junio del año siguiente, con dos sesiones semanales de una hora de duración. La práctica deportiva se realizó en un campo de fútbol 11 con el equipamiento necesario. En estas sesiones se llevaron a cabo diferentes ejercicios, actividades y circuitos, que sirvieron para modelar las diferentes habilidades necesarias para aprender a desarrollar esta actividad, o para mejorar la ejecución de una habilidad ya adquirida.

Además, la adaptación y supervisión de este programa se llevó a cabo por un equipo multidisciplinar compuesto por terapeutas ocupacionales, psicólogos, maestros de educación física y entrenadores.

\section{Instrumentos}

Se evaluó el nivel inicial de las variables en las que se esperaba un cambio tanto antes como después de llevarse a cabo el programa, evaluando así los cambios en estas variables. Se evaluó de manera independiente, por un lado, el desarrollo físico, y por otro las habilidades sociales.

Para evaluar el desarrollo físico se tomó como referencia los Estándares de Aprendizaje para el área de Educación Física, contemplados en la etapa de Educación Primaria por la actual ley educativa, la Ley Orgánica para la Mejora de la Calidad Educativa (Ley No 295, 2013).

Los Estándares de Aprendizaje seleccionados para este estudio fueron aquellos que influían directamente en el desempeño motor de los niños. Además, se han incluido aquellos valores y actitudes referenciados al cuerpo, al movimiento y a la relación de los participantes con el entorno, ya que carece de sentido tener en cuenta la práctica deportiva sin comprender aquellos aspectos que dan sentido a las acciones motrices.

Los ítems elegidos para evaluar a cada uno de los particiTabla 2

\begin{tabular}{|c|c|c|c|}
\hline \\
\hline 1 & $\begin{array}{l}\text { Habilidades motriz } \\
\text { básicas }\end{array}$ & \multicolumn{2}{|c|}{$\begin{array}{l}\text { Descripción del constructo } \\
\text { El desplazamiento, el salto, el lanzamiento, la recepción y } \\
\text { el giro. }\end{array}$} \\
\hline 2 & $\begin{array}{l}\text { Habilidades motriz } \\
\text { avanzadas }\end{array}$ & \multicolumn{2}{|c|}{ El pase, la conducción, el tiro y el control. } \\
\hline 3 & $\begin{array}{l}\text { Aspectos vinculados a } \\
\text { la cooperación }\end{array}$ & \multicolumn{2}{|c|}{ La recogida y organización del material. } \\
\hline \multicolumn{4}{|c|}{$\begin{array}{l}\text { Tabla } 3 \\
\text { Valores de puntuación de la escala Likert }\end{array}$} \\
\hline \multicolumn{3}{|c|}{ Descriptor } & Valor numérico \\
\hline \multicolumn{3}{|c|}{ Realiza la acción, sin errores y de forma espontánea. } & 5 \\
\hline \multicolumn{3}{|c|}{ Realiza la acción, con pocos errores y de forma espontánea. } & 4 \\
\hline \multicolumn{3}{|c|}{ Realiza la acción, pero con algunos errores y no de forma espontánea. } & 3 \\
\hline \multicolumn{3}{|c|}{ Realiza la acción, pero con muchos errores y no de forma espontánea. } & 2 \\
\hline \multicolumn{3}{|c|}{$\begin{array}{l}\text { Realiza la acción, pero con muchos errores y no de forma espontánea. } \\
\text { No realiza la acción en absoluto. }\end{array}$} & \\
\hline
\end{tabular}

pantes aparecen en la tabla 2.

Estos ítems se evaluaron de manera cualitativa a través del sistema de rúbrica, mediante la observación en cada una de las sesiones que han tenido lugar, desde el comienzo del programa hasta su finalización. En cada sesión había dos observadores entrenados para tal fin que contaban con la rúbrica anteriormente mencionada. Además de la comparación de las anotaciones y observaciones de ambos observadores, se tuvo en consideración la opinión de los entrenadores a lo largo de las sesiones. Los ítems se han evaluado mediante una escala de tipo Likert, con puntuaciones comprendidas entre 1 y 5 , siendo 1 la peor calificación y 5 la mejor calificación, distribuidas en la Tabla 3.

Para la evaluación de habilidades sociales se partió del modelo de habilidades sociales de Goldstein (Goldstein, Sprafkin, Gershaw \& Klein, 1980) adaptando los seis bloques que conforman su modelo a cuatro, que son los que se van a evaluar. Esta decisión se ha realizado en función de las actividades que se desarrollaron durante los ocho meses que duró el programa de actividades físicas. De este modo, algunos de los ítems desaparecieron puesto que no iban a ser trabajados o no se adecuaban a las sesiones organizadas. Los bloques con los ítems evaluados fueron los siguientes (ver tabla 3):

\section{Resultados}

A continuación, se expone en primer lugar los resultados de la evaluación del desarrollo motor, y posteriormente, se presentan los resultados de la evaluación en habilidades sociales en bloques, tal y como se describe en la tabla 2 y en la tabla 4. Para analizar los resultados se ha optado por realizar la prueba no paramétrica de Wilcoxon para muestras emparejadas, debido al reducido tamaño muestral. Los resultados muestran diferencias significativas entre las puntuaciones observadas en el mes de noviembre, al inicio del programa, y en el mes de junio, al finalizarlo. Además, se han realizado comparaciones entre las puntuaciones medias obtenidas periódicamente a lo largo de todo el programa. Estos se proyectan en las figura 1, figura 2 y figura 3 , dando lugar a gráficos que muestran la evolución de los participantes.

Tabla 4

Descripción de las variables de habilidades sociales evaluadas.

Bloques Constructo evaluado Descripción del constructo

1 Habilidades sociales primarias* Presta atención, inicia conversación, mantiene conversación, formula preguntas, se presenta, da las \begin{tabular}{lll} 
& & gracias y saluda. \\
\hline 2 & Habilidades sociales & Pide ayuda, participa en las actividades, sigue
\end{tabular} \begin{tabular}{lll} 
avanzadas** & instrucciones, da instrucciones y discute. \\
\hline 3 & Habilidades relacionadas con losReconoce y expresa sus sentimientos, expresa afecto,
\end{tabular} Habilidades relacionadas con los Reconoce y expresa sus sentimientos, expresa afecto,
sentimientos**
comprende sentimientos ajenos y controla la rabia. 4 Habilidades sociales $\quad$ Formula quejas, responde bien a quejas, responde ante relacionadas con el acusaciones, resuelve la timidez, ayuda a otros, comportamiento** presenta deportividad tras el juego y responde a la $\frac{\text { persuasion. }}{\text { Nota. Basado en Goldstein et al. (1980). }{ }^{*} \text { En el bloque uno, los ítems se evaluaron de forma }}$ dicotómica, estando los valores comprendidos entre 0 (no lo hace) y 1 (sí lo hace), por lo que un pequeño cambio en la media de las respuestas podría parecer un gran cambio en los comportamientos de los jugadores. ** Los ítems de los bloques dos, tres y cuatro, fueron evaluados mediante escala tipo Likert, con una puntuación de 1 a 5 , donde 1 era la peor respuesta y 5 la respuesta correcta (ver Tabla 3 ).

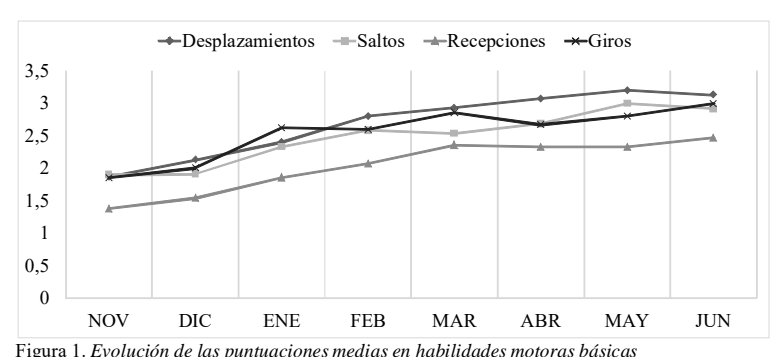

Figura 1. Evolución de las puntuaciones medias en habilidades motoras básicas

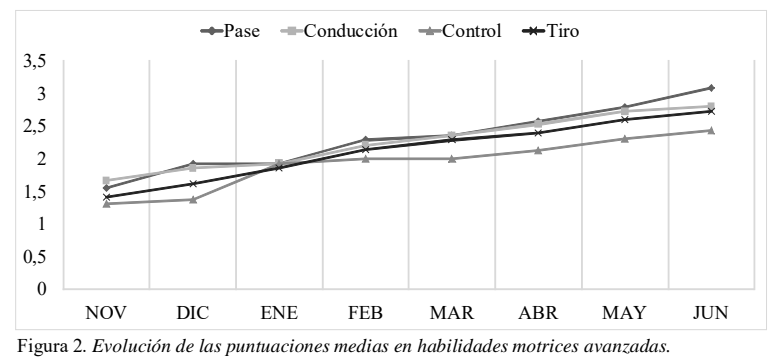




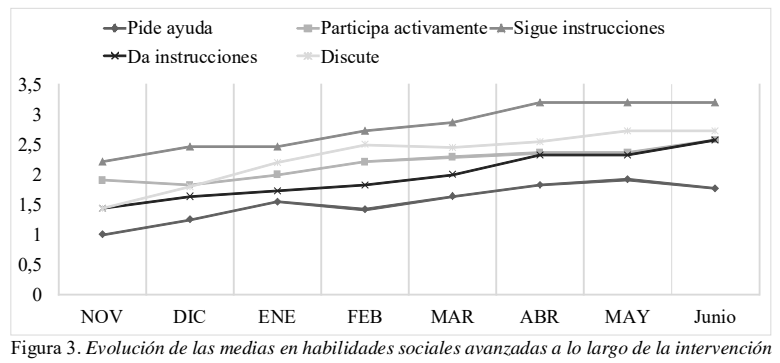

\section{Desarrollo motor}

En este ámbito de trabajo se adaptaron situaciones motrices con diversidad de estímulos y condicionantes espaciotemporales, seleccionando y combinando las habilidades motrices básicas y adaptándolas a las condiciones de forma eficaz.

En general, la consecución de los ítems para este bloque fue favorable. La mejora más clara en la ejecución de las actividades se dio en los meses de noviembre y diciembre y en enero. Cabe destacar que en el mes de enero se produjo un breve estancamiento en cuanto a la adquisición de habilidades motrices. Esta regresión pareció repetirse a lo largo de toda la evaluación, tanto a nivel motriz, social y de comunicación.

A la hora de explicar los resultados se han tenido en cuenta las observaciones mensuales que se llevaron a cabo a lo largo de todo el programa, con inicio en noviembre y finalización en junio. Con los datos obtenidos en noviembre se pudo establecer una línea base que supuso el punto de partida de la investigación. Los datos del periodo de marzo aportaron un conocimiento global de la evolución siendo este una aproximación a la mitad del programa. Por último, con los datos obtenidos en junio se pudo obtener el resultado final del progreso de los participantes, tal y como se puede observar en las siguientes líneas.

En cuanto a las habilidades motrices básicas, las actividades de recepción fueron las que más dificultad entrañaron para los niños, mientras que en desplazamiento, salto y giro obtuvieron mejores resultados (ver figura 1). Todas las actividades que se organizaron llevaban implícitos retos técnico-tácticos elementales propios del juego y actividades físicas con o sin oposición aplicando principios y reglas para resolver las situaciones motrices actuando de forma individual coordinada y cooperativa y desempeñando las diferentes funciones implícitas en juegos y actividades. Por su parte, atendiendo a las habilidades motrices avanzadas, concretamente el pase y la conducción fueron las habilidades que mejor se adquirieron, siendo logradas con puntuaciones medias elevadas por todos los participantes. El tiro de pelota también fue conseguido satisfactoriamente, mientras que el control requería de más entrenamiento para ser mejorado (ver figura 2).

De cualquier modo, la mayor parte de los niños mostraron un gran interés por mejorar sus habilidades, a la vez que adquirieron cierta autonomía y confianza en distintas situaciones. Por último, los niños aprendieron sin problema la rutina de recoger el material utilizado durante las sesiones como parte del proceso de aprendizaje. Además, tal y como se muestra en la tabla 5 , tabla 6 y tabla 7 , se han producido cambios significativos en todos los ítems analizados anteriormente.
Prueba de Wilcoxon en desarrollo motriz básico

\begin{tabular}{lcc} 
& \\
\hline Pruesarrollo motriz básico & Puntuación Z & Significación \\
\hline Desplazamientos & -3.27 & .001 \\
Saltos & -2.88 & .004 \\
Recepciones & -3.17 & .002 \\
Giros & -3.14 & .002 \\
\hline \multicolumn{2}{l}{ Tabla 6 } & \\
Prueba de Wilcoxon en desarrollo motriz avanzado & \\
\hline Desarrollo motriz avanzado & Puntuación Z & Significación \\
\hline Pase del balón & -2.86 & .004 \\
Conducción del balón & -2.74 & .006 \\
Tiros con balón & -3.14 & .002 \\
Control del balón & -3.17 & .001 \\
\hline
\end{tabular}

Tabla 7

Prueba de Wilcoxon en desarrollo cooperativo

Desarrollo cooperativo Puntuación $Z$ Recoge y organiza

\section{Desarrollo social}

En lo referente al apartado de desarrollo social, el fútbol, al ser un deporte colectivo, el propio contexto de las actividades traía consigo Oportunidades de interacción social. En mayor o menor medida, se observó una mejora significativa en casi todas las variables.

Los datos relativos al bloque de habilidades sociales primarias muestran que, en la mayoría de los casos, la capacidad atencional partía de puntuaciones bajas, que mejoraron muy discretamente y no de forma significativa, como se indica en la tabla 6 , al tener una significación superior a .05 ( $p$ $>.05$ ). Aunque en un principio, sí que presentaban dificultades para mantener el nivel de atención tanto en las explicaciones como en la ejecución de las tareas. Posteriormente, todos seguían las instrucciones del entrenador precisando elevado nivel de apoyo a la hora de realizar actividades, como por ejemplo, mantener una fila o respetar el orden de actuación.

La mayoría de los participantes desarrollaron la capacidad de iniciar conversaciones y mantenerlas mostrando algunas peculiaridades, como por ejemplo, cambios de tema drásticos, incongruencia en algunas afirmaciones o desestructuración del lenguaje, entre otras. La capacidad para formular de preguntas se manifestaba en todos los participantes, exceptuando a cinco, que mostraban un nivel de adquisición del lenguaje más bajo atendiendo a su edad cronológica. Del mismo modo, 12 de los participantes mejoraron su capacidad para presentarse ante una persona desconocida y casi todos, aprendieron a dar las gracias con correspondencia. Asimismo, la mayoría de los participantes adquirieron el hábito del saludo; aunque dos de ellos, en ocasiones, precisaban apoyo y lo hacían mediante comuni-

\begin{tabular}{lcc}
$\begin{array}{l}\text { Tabla } 8 \\
\text { Prueba de Wilcoxon en habilidades sociales primarias }\end{array}$ & \\
\hline Habilidad Social & Puntuación Z & Significación \\
\hline Presta atención & -1.41 & .157 \\
Inicia conversación & -3.32 & .001 \\
Mantiene conversación & -2.83 & .005 \\
Formula preguntas & -2.64 & .008 \\
Se presenta & -2.64 & .008 \\
Agradece & -3.32 & .001 \\
Saluda & -3.16 & .002 \\
\hline & & \\
Tabla 9 & & \\
Prueba de Wilcoxon en habilidades sociales avanzadas & Significación \\
\hline Habilidad Social & -1.86 & .063 \\
\hline Pide ayuda & -1.81 & .070 \\
Participa activamente & -3.13 & .001 \\
Sigue instrucciones & -2.31 & .005 \\
Da instrucciones & -2.71 & .003 \\
Discute & & \\
\hline & & \\
Tabla 10 & \multicolumn{2}{l}{ Prueba de Wilcoxon en habilidades sociales relacionadas con el comportamiento } \\
\hline Habilidad Social & Puntuación Z & Significación \\
\hline Pide permiso & -2.53 & .011 \\
Autocontrol & -2.89 & .004 \\
Formula quejas & -2.76 & .006 \\
Responde ante acusación & -2.92 & .004 \\
Ayuda y defiende a otros & -1.89 & .059 \\
\hline
\end{tabular}


cación no verbal (ver tabla 8).

Haciendo alusión a las habilidades sociales avanzadas, se observó que los participantes no solicitaban ayuda de forma independiente. No obstante, se mostraban frustrados en las situaciones en las que no realizan un ejercicio de forma autónoma. Por otro lado, no se encuentran diferencias significativas en las variables «petición de ayuda» y «participación activa en las actividades», tal y como se puede apreciar en la tabla 9. La figura 3 muestra un incremento progresivo a lo largo de todo el programa en las habilidades sociales avanzadas de todos los participantes.

Por su parte, el abordaje de las habilidades sociales relacionadas con los sentimientos no fue posible en este contexto, puesto que no tuvieron lugar situaciones diarias en las que se pudiese trabajar las emociones. En cuanto a las habilidades sociales relacionadas con el comportamiento, se observaron mejoras significativas en la mayor parte de la muestra (ver tabla 10). También se mostraron notables mejoras en la capacidad de autocontrol de todos los sujetos, salvo una excepción, donde mostró un comportamiento agresivo entre compañeros, hacia los entrenadores y hacia los propios padres. Tan solo tres de los participantes fueron capaces de llegar a elaborar quejas de forma correcta. En cuanto a la respuesta ante acusaciones, cuatro de los niños comenzaron a responder correctamente; aunque, en ocasiones, las acusaciones resultaban ser una broma que aceptaban con buen humor y sin llegar a molestarse. Salvo en alguna ocasión, no se mostró una predisposición a ayudar o defender a otros compañeros.

\section{Discusión}

En general, la implementación del programa deportivo basado en la actividad física del fútbol ha supuesto una mejora tanto en las habilidades motrices como en las habilidades sociales. Sin embargo, en el último tramo del programa se ha observado una tendencia a un rendimiento inferior. Esto puede deberse al número de sesiones y la duración de cada una de ellas, lo que implica una reducción de la atención y del interés por la actividad (Camacho, 2019). Para evitar esta situación se recomienda disminuir la duración total del programa, e incluso de las sesiones, con el fin de evaluar si persiste esta tendencia a la reducción de rendimiento por el hecho de ser una actividad de larga duración (Camacho, 2019). Por otro lado, el esfuerzo mental al que está sometido el jugador debido a las posibles alternativas de acción en el juego puede reducir los recursos del sistema cognitivo, causando aparición de fatiga e influyendo negativamente en el rendimiento (Camacho, Caraballo \& Alías, 2019).

El proceso de aprendizaje en el fútbol requiere de una serie de fases de adquisición de conocimientos. Es decir, dependiendo de la edad o del nivel de conocimientos y experiencias del que parte el jugador, habrá habilidades que no se podrán evaluar del mismo modo, bien por ser más complejas o porque requieran de una combinación de varias habilidades motrices (Figueredo, Figueredo \& Jiménez, 2019). Dicho esto, no es casualidad que los niños puedan presentar dificultades en el desarrollo de algunas habilidades deportivas frente a otras, pues la etapa de formación debe ser entendida como un proceso gradual, continuo y debidamente organi- zado (Wein, 1995 y Garganta, 2002), además de tener en cuenta las características psicoevolutivas de estos (Giménez, 2000; Lillo, 2000 y Lago, 2001).

La metodología del entrenamiento y del juego, junto con la continua repetición de las tareas, supuso una mejora en el rendimiento deportivo provocando así, una motivación intrínseca en el jugador. Esto aumentó el interés en la participación, y sirvió para adquirir una mayor confianza y autonomía para resolver ciertas situaciones (Arroyo, 2019). Respecto al ámbito de las habilidades sociales, se hacía evidente el trabajo previo realizado por parte de las familias y los centros educativos con los niños TEA. Si bien, algunos presentaron dificultades de interacción al inicio del programa. El clima de confianza que se generó hizo que se produjese una mejora general, pero principalmente, en las habilidades sociales primarias y avanzadas (Hollews et al., 2020).

Por su parte, las dificultades para mantener el nivel de atención durante las explicaciones así como en la ejecución de las tareas no fueron notables. Incluso, se observaron mejoras en el mantenimiento de la atención a largo plazo. Asimismo, se redujo considerablemente el número de errores que cometían a la par que aprendían a realizar los ejercicios. En líneas generales, esto puede deberse a que las instrucciones eran sencillas y, por tanto, se mejoraba el aprendizaje y la retención de las instrucciones para la ejecución de una habilidad deportiva (Camacho, 2019).

Haciendo referencia a las habilidades sociales relacionadas con el comportamiento, en concreto, las relacionadas con las escasas situaciones en las que se dieron conductas agresivas, se observó una mejora una vez que las familias contribuyeron en su resolución desde el contexto familiar en colaboración con el contexto educativo y el propio del programa deportivo. Varios estudios demuestran que la colaboración de las familias en el proceso de aprendizaje mejora las interacciones sociales entre pares (Arroyo, 2019; Healy et al., 2018; Holland et al., 2019). Además, se observó también que, bajo instrucción, son capaces de pedir disculpas y abandonar la pelea, lo cual es un aspecto muy positivo.

Respecto a las habilidades sociales relacionadas con las emociones, no se ha podido trabajar de manera específica ya que no se han dado las situaciones necesarias o, al menos, no con la frecuencia suficiente como para poder ser evaluadas de forma similar al resto de variables. La propuesta de talleres específicos donde se puedan establecer escenarios controlados para poder evaluar las habilidades sociales relacionadas con las emocionales, cobra especial importancia ante este escenario.

Las situaciones en las que se pudieran emitir quejas no se daban a menudo, pero cuando se daban, los niños hacían evidente su frustración y formulaban quejas razonables.

En lo relativo a la respuesta ante acusaciones, es probable que se tratara de una conducta aprendida, pues resultaba evidente que no comprendían la broma y la sonrisa era, en ocasiones, forzada. De acuerdo con Agius y Levey (2019), el sentido del humor funciona como un pegamento social, por lo que las intervenciones que consisten en el entrenamiento del humor tienen gran importancia para las personas con TEA.

Tomando como base los resultados obtenidos, en un contexto de mejora generalizada en el ámbito físico/motriz de 
los participantes, así como en el ámbito social/comunicativo, es posible que el desarrollo de programas socio-deportivos similares tengan un impacto positivo en personas con TEA. Además, estos programas pueden llevarse a cabo en las instalaciones de ámbitos familiares para los niños: los colegios o institutos, en los que se sienten seguros y cómodos, ya que son entornos conocidos y que han tenido la oportunidad de explorar en múltiples ocasiones. En investigaciones similares, se han descrito experiencias sociales positivas, facilitando el acceso al deporte y a la interacción, de modo que también se fomenta el desarrollo del deporte inclusivo (Ryan, S., 2019)

\section{Conclusiones}

El eje principal sobre el que se sustenta la investigación es el desarrollo de un programa deportivo que pueda servir para entrenar, de forma simultánea, el desarrollo motriz y las habilidades sociales de niños con trastorno del espectro del autismo. De forma específica, también se persigue estudiar las repercusiones que tiene la práctica deportiva en niños con trastorno del espectro del autismo en un entorno controlado.

En términos generales, los resultados obtenidos tras la ejecución de este programa, cumplen las expectativas previstas. Se ha logrado una mejora generalizada y significativa del rendimiento físico de los participantes y de la mayor parte de las habilidades sociales observadas.

En lo relativo a las habilidades motrices, la actividad física y deportiva ha tenido un beneficio y un impacto positivo sobre los participantes, pues se ha podido observar un aumento eficaz en la capacidad de movimiento y de interacción con el entorno.

Algunas de las habilidades sociales descritas por Goldstein (1980) no pudieron ser evaluadas, por lo que el programa puede necesitar algunos ajustes, con la finalidad de establecer intervenciones más específicas y destinadas a trabajar aquellos aspectos que no han podido ser abordados.

Se considera importante, de cara a futuras investigaciones, que se exploren otras variables que pueden afectar al desarrollo de los participantes, tales como la modalidad de escolarización y la participación en otros programas o actividades que puedan influir en los resultados obtenidos.

Además, el desarrollo del curso ha otorgado distintos beneficios para la vida cotidiana de los alumnos, pues se han trabajado competencias transversales como la espera en una fila, la discriminación de estímulos, y las habilidades motrices necesarias para la interacción con el resto de niños, permitiéndoles así generalizar los juegos en otros lugares de ocio.

Se ha podido observar una tendencia a la reducción del rendimiento en algunos ítems observados en el último mes de duración del programa. En futuras ocasiones, puede resultar interesante observar este efecto, reduciendo la duración total del programa y de la duración de las sesiones, tratando de evitar que el participante sienta cansancio o agotamiento que pueda afectar negativamente a su bienestar y rendimiento.

Otra propuesta de mejora, puede ser el uso de otras actividades deportivas inclusivas y cooperativas. Por ejemplo, el Colpbol, deporte en el que necesariamente han de participar todos los miembros del equipo, fomentando la motivación y la interacción, tal y como describen, Hernández, Martínez y Carrión (2019).

\section{Referencias}

Agius, J. \& Levey, S. (2019). Humour and Autism Spectrum Disoreders. Malta Journal of Health Sciences, 6(1), 2228. doi: 10.14614/HumourAUTISM/10/19

American Psychiatric Association. (2013). Diagnostic and Statistical Manual of Mental Disorders, 5th Edition (DSM-5). doi: 10.1176/appi.books.9780890425596.744053

Arroyo, R. (2019). Effective ways of teaching children with autism spectrum disorder in inclusive physical education setting. Kinesiology, Sport Studies and Physical Education, 81. Recuperado de https:// digitalcommons.brockport.edu/pes_synthesis/81/

Camacho, P. (2019). Efecto del foco atencional sobre el aprendizaje de las habilidades deportivas individuales. Retos, 36, 561-566. Recuperado de https://recyt.fecyt.es/ index.php/retos/article/view/64428

Camacho, P., Caraballo, I., Alías, A. (2019). Efecto de las estrategias de enseñanza incidentales sobre la carga de trabajo en el fútbol. Una revisión sistemática. Journal of Sport and Health Research, 11(3), 211-226. Recuperado de http:/www.journalshr.com/index.php/issues/2019

Cei, A., Franceschi, P., Rosci, M., Sepio, D. \& Ruscello, B. (2017). Motor and psychosocial development in children with autism spectrum disorder through soccer. International Journal of Sport Psychology, 48(5), 485507. doi: 10.7352/IJSP.2017.48.485

Cos, F., Cos, M. Á., Buenaventura, L., Pruna, R. \& Ekstrand, J. (2010). Modelos de análisis para la prevención de lesiones en el deporte. Estudio epidemiológico de lesiones: el modelo Union of European Football Associations en el fútbol. Apunts. Medicina de l'Esport, 45(166), 95102. doi: 10.1016/j.apunts.2010.02.007

Díez, A., Muñoz, J. A., Fuentes, J., Canal, R., Idiazábal, M. A, Palacios, S., Sanidad, D. (2005). Guía de buena práctica para el diagnóstico de los trastornos del espectro autista. Revista neurológica, 41(5), 299-310. doi:10.4321/S113014732009000400001

Figueredo, L., Figueredo, E. \& Jiménez, R. (2019). Libro: actividades físicas para niños autistas. Una metodología para su atención. Revista de la Facultad de Cultura Física de la Universidad de Granma, 16(54), 229-239. Recuperado de https://revistas.udg.co.cu/index.php/olimpia/ article/view/720

Fuentes, J., Ferrari, M. J., Boada, L., Touriño, E., Martos, J., Posada, M., (2006). Guía de buena práctica para el tratamiento de los trastornos del espectro autista. Revista de Neurología, 43(7), 425-438. doi: 10.33588/rn.4307.2005750

Garganta, J. (2002). Competências no ensino e treino de jovens futebolistas. Lecturas: Educación Física y Deportes, 45. Recuperado de https://www.efdeportes.com/ efd45/ensino.htm

Giménez, F. J. (2000). Fases en la formación del deportista y su aplicación en la iniciación deportiva. Habilidad Motriz. Revista de Ciencias de la Actividad Física y del Deporte (15), 35-39. Recuperado de https://1c501c57-fd274 abb-af5a-54a 74 c 8731 d2 .filesusr.com/ugd/ 28d333_5f294f1c9ed44ac1a3a96f4cff17e803.pdf 
Goldstein, A. P., Sprafkin, R. P., Gershaw, N. J. \& Klein, P. (1980). Adolescent: social skills training through structured learning. Teaching social skills to children: innovative approaches. Edited by Gwendolyn Cartledge and JoAnne Fellows Milburn. Recuperado de http:// a g r i s.f a o.o r g/a g r i s - s e a r c h/ search.do?recordID=US201302643959

Gutiérrez, M. (2004). El valor del deporte en la educación integral del ser humano. Revista de educación, (335), 105-126. Recuperado de http:// www.educacionyfp.gob.es/revista-de-educacion/ numeros-revista-educacion/numeros-anteriores/2004/ re335/re335-09.html

Harris, S. (2017). Early motor delays as diagnostic clues in autism spectrum disorder. European Journal of Pediatrics, 176, 1259-1262. doi: 10.1007/s00431-017-29517

Healy, S., Nacario, A., Braithwaite, R. \& Hopper, C. (2018). The effect of physical activity interventions on youth with autism spectrum disorder: a meta-analysis. Autism Research, 11(6), 1-16. doi: 10.1002/aur.1955

Hernándea, A., Martínez, I. y Carrión, S. (2019). El Colpbol como un medio para incrementar la motivación en Educación Primaria. Retos, 36, 348-353. Recuperado de https:/ /recyt.fecyt.es/index.php/retos/article/view/70396

Holland, S., Holland, K., Haegele, J. \& Alber-Morgan, S. (2019). Making it stick: teaching students with autism to generalize physical education skills. Journal of Physical Education, Recreation \& Dance, 90(6), 32-39. doi: 10.1080/07303084.2019.1614120

Howells, K., Sivaratman, C., Lindor, T., Hyde, C., McGillivray, J., Whithouse, A. \& Rinehart, N. (2020). Can participation in a community organized football program improve social, behavioural functioning and communication in children with autism spectrum disorder? A pilot study. Journal of Autism and Development Disorders. doi: 10.1007/s10803-020-04423-5

Howells, K., Sivaratnam, C., May, T., Lindor, E., McGillivray, J., \& Rinehart, N. (2019). Efficacy of group-based organised physical activity participation for social outcomes in children with autism spectrum disorder: A systematic review and meta-analysis. Journal of Autism \& Developmental Disorders, 49(8), 3290-3308. doi:10.1007/s1080 3-019-04050-9

Ibáñez, P. \& Mudarra, M. J. (2004). Relaciones sociales de personas con discapacidad, en el Ocio y Tiempo libre. Revista Española de Pedagogía, 229, 521-540. Recuperado de https://revistadepedagogia.org/lxii/no-229/relaciones-sociales-de-personas-con-discapacidad-en-elocio-y-tiempo-libre/101400010496/

Kruger, G., Silveira, S. \& Marques, A. (2019). Motor skills of children with autism spectrum disorder. Revista Brasileira de Cineantropometria y Desempenho $\mathrm{Hu}$ mano, 21, 1-8. doi:10.1590/1980-0037.2019v21e60515

Lago, C. (2001). El proceso de iniciación deportiva en el fútbol, secuenciación de los contenidos técnico-tácticos. Training fútbol: revista técnica del fútbol (66), 34-45. Recuperado de http://www.trainingfutbol.com/

Ley Orgánica No 295. Boletín Oficial del Estado, España, de 10 de diciembre de 2013.

Lillo, J. M. (2000). Consideraciones de aplicación al entrenamiento de la táctica. Training fútbol: revista técnica del fútbol (47), 8-13. Recuperado de http:// www.trainingfutbol.com/

May, T., Rinehart, N., Barnett, L., Hinkley, T., McGillivray, J. \& Skouteris, H. (2018). We're doing AFLAuskick as well': experiences of an adapted football program for children with autism. Journal of Motor Learning and Development,6, 130-146. doi: 10.1123/jmld.2016-0055

Merino, A., Jarie, L. \& Usán, P. (2019). Referentes formativodeportivos en el fútbol base español: un escenario socioeducativo complejo. Educación Física y Ciencia, 21(2), e078. doi: 10.24215/23142561e 078

Monjas, R., Ponce, A. \& Gea, J. M. (2015). La transmisión de valores a través del deporte: deporte escolar y deporte federado. Retos, 28, 276-284. Recuperado de https:// recyt.fecyt.es/index.php/retos/article/view/35650/19675

Núñez, C. R. G. (2005). Habilidades sociales, clima social familiar y rendimiento académico en estudiantes universitarios. Liberabit: Revista peruana de psicología, 11(11), 63-74. Recuperado de:

http://ojs3.revistaliberabit.com/publicaciones/ ?portfolio=revista-liberabit-vol-11

Obrusnikova, I. \& Cavalier, A. R. (2010). Perceived Barriers and Facilitators of Participation in After-School Physical Activity by Children with Autism Spectrum Disorders. Journal of Developmental and Physical Disabilities, 23(3), 195-211. doi:10.1007/s10882-010-9215-Z

Ohara, R., Kanejima, Y., Kitamura, M. \& Izawa, K. P. (2020). Association between social skills and motor skills in individuals with autism spectrum disorder: a systematic review. European Journal of Investigation in Health, Psychology and Education, 10, 276-296. doi:10.3390/ ejihpe10010022

Pan, C.Y. (2008). Objectively measured physical activity between children with autism spectrum disorders and children without disabilities during inclusive recess settings in Taiwan. Journal of autism and developmental disorders, 38(7), 1292-301. doi:10.1007/s10803-007-05186

Reinders, N., Branco, A., Wright, K., Fletcher, P. \& Bryden, P. (2019). Scoping review: physical activity and social functioning in young people with autism spectrum disorder. Frontiers in Psychology, 10(120), 1-17. doi: 10.3389/fpsyg.2019.00120

Ruiz, D., Salinero, J. J., González, C., Lledó, M., García, T., Theirs, C. I., Melero, D. \& Guitián, A. (2015). Descripción de la práctica de actividad física, habilidades motrices básicas y composición corporal en niños y jóvenes de espectro autista. Diferencias por sexo. Retos, 28 (61-65).

Ryan, S. (2019). Sport involvement for youth with autism spectrum disorders and intellectual disabilities (Tesis doctoral). York university, Toronto.

Smith, T., Scahill, L., Dawson, G., Guthrie, D., Lord, C., Odom, S., Rogers, S. \& Wagner, A. (2007). Designing research studies on psychosocial interventions in autism. Journal of Autism and Developmental Disorders, 37(2), 354-366. doi:10.1007/s10803-006-0173-3.

Wein, H. (1995). Fútbol a la medida del niño. Madrid: Real Federación Española de Fútbol.

Wong, C., Odom, S., Hume, K., Cox, A., Fettig, A., Kucharrczyk, S., Brock, M., Plavnick, J., Fleury, V. \& Schultz, T. (2015). Evidence based practices for children, youth, and young adults with autism spectrum disorder: a comprehensive review. Journal of Autism and Developmental Disorders, 45, 1951-1966. doi: 10.1007/ s10803-014-2351-z 